\title{
Diabetic retinopathy, diabetic macular edema, and cardiovascular risk: the importance of a long-term perspective and a multidisciplinary approach to optimal intravitreal therapy
}

\author{
Francesco Bandello ${ }^{1}$ (D) . Danilo Toni ${ }^{2} \cdot$ Massimo Porta $^{3} \cdot$ Monica Varano $^{4}$
}

Received: 9 September 2019 / Accepted: 5 November 2019 / Published online: 20 November 2019

(c) Springer-Verlag Italia S.r.l., part of Springer Nature 2019

\begin{abstract}
Diabetic retinopathy (DR), diabetic macular edema (DME), and cardiovascular disease (CVD) resulting from vascular damage from persistently elevated blood glucose levels are among the serious secondary pathologies associated with long-standing diabetes mellitus. The established link between DR and CVD suggests the need for appropriate and early management of patients with diabetes to minimize CV risk. This is of particular importance in patients with recent, or a history of, major CV events. Early management of DR is a complex task that requires comprehensive evaluation and a multidisciplinary approach to manage complications, risk factors, and interactions between different aspects of the disease. Anti-vascular endothelial growth factor (VEGF) agents have become an important therapeutic modality in ophthalmology. However, their use is contraindicated in patients with DR and/or DME with a CV event in the previous 3 months. In patients with DME, corticosteroids target the multifaceted inflammatory pathways involved in the pathogenesis of DR, with a broader spectrum of action than anti-VEGF agents. In this context, recent guidelines suggest the use of corticosteroids, and in particular dexamethasone intravitreal implant, as a well-tolerated and efficacious first-line treatment in patients with high CV risk, such as a history of or recent major CV events. This review focuses on the subset of diabetic patients with a prior CV event, DR, and DME and discusses the need for a holistic approach in evaluating the optimal therapeutic choice for the care of the individual patient, supported by real-world clinical experience on long-term dexamethasone intravitreal implant therapy.
\end{abstract}

Keywords Diabetic retinopathy (DR) - Diabetic macular edema (DME) - Anti-VEGF (vascular endothelial growth factor) agents · Corticosteroids · Cardiovascular (CV) risk

\section{Introduction}

Diabetes mellitus is a chronic and progressive metabolic disease due to absolute (Type 1) or relative (Type 2) insulin deficiency causing hyperglycemia [1]. Recent epidemiological data from the International Diabetes Federation (IDF) estimate that in 2017 , the global prevalence of diabetes was 8.8\% among individuals 20-79 years of age, and projected to increase to $9.9 \%$ by 2045 [1]. Most cases (87-91\%) are represented by type 2 diabetes, with overweight and obesity being the main risk factors $[1,2]$.

Long-standing diabetes is associated with a range of secondary pathologies due to vascular damage from persistently elevated blood glucose levels, which include both macrovascular [coronary artery disease (CAD), peripheral artery disease (PAD), and cerebrovascular disease] and microvascular [peripheral neuropathy, diabetic eye disease mainly cataracts, diabetic retinopathy (DR), and diabetic 
macular edema (DME)] complications. Given this, the risk of cardiovascular (CV) disease is increased two-threefold in patients with diabetes [1] and is comparable to the risk of non-diabetes subjects with a previous CV accident [3, 4]. In fact, a study performed in the Finnish population found that the incidence of myocardial infarction (MI) over 7 years in subjects with type 2 diabetes (20.2\%) was highly similar to that of non-diabetes subjects with previous MI (18.8\%) [3]. In a later study in the Danish population of at least 30 years of age, patients with diabetes had a risk of CV mortality that was comparable to non-diabetic patients suffering from a previous MI, with no correlation between sex and type of diabetes [4].

Diabetes-related complications are thus associated with increased mortality, and in 2016, diabetes was identified as the seventh leading cause of death worldwide [5]. In fact, mortality in patients with type 2 diabetes is about threefold higher than in healthy individuals, mainly due to $\mathrm{CV}$ and renal complications [6]. For example, in the Verona Diabetes Study, CV disease accounted for $41.8 \%$ of deaths in the study population [6]. More recently, it has been estimated that $65 \%$ of deaths due to diabetes in the USA are due to CV disease, stroke, or both $[1,6,7]$. Therefore, in the case of $\mathrm{CV}$ events, there is an objective need for prolonged surveillance (beyond the first year), particularly in patients with additional risk factors such as diabetes.

Considering the above, the need for appropriate and early management of $\mathrm{CV}$ risk in patients with diabetes has recently been reinforced by guidelines from the European Society of Cardiology [8] and the American Diabetes Association [9, 10]. In addition, another important aspect of diabetes management is related to the strong correlation between microvascular and macrovascular complications, and, in this regard, the presence of DR has been associated with the development of $\mathrm{CV}$ disease, cerebrovascular disease, and PAD [11].

Early management requires comprehensive evaluation and a multidisciplinary approach to manage complications, risk factors, and interactions between different aspects of the disease. The present review will focus on the subset of diabetic patients with a prior CV event, DR, and DME as an example of such interactions and will discuss the need for a holistic approach in evaluating the optimal therapeutic choice for the care of the individual patient. In particular, potential differences in individual systemic absorption after intravitreal drug administration and the subsequent impact that such alterations may have in both the short- and long term will be considered.

\section{Diabetic eye disease and cardiovascular risk}

Diabetic eye disease is, in fact, a spectrum of ocular pathologies, ranging from DR and DME to cataracts, glaucoma, and double vision [1]. Signs of DR can be identified in about onethird of patients with diabetes [12], and it is a leading cause of blindness in the working-age population in developed countries $[1,10,13]$. Vision-threatening states, such as DME and proliferative retinopathy, are recognized in about one-tenth of the diabetic population $[14,15]$. While proliferative retinopathy is mainly characterized by neovascularization due to retinal hypoxia, DME involves disruption of the blood-retinal barrier (BRB), leading to fluid accumulation in the central part of the retina [16].

From a pathophysiological standpoint, inflammation plays a central role in the pathogenesis of DR, especially for DME [16]. At the same time, hyperglycemia, advanced glycation end products (AGEs), proinflammatory cytokines, and retinal hypoxia are all responsible for upregulation of vascular endothelial growth factor (VEGF), which leads to alterations in the blood-retinal barrier (through increased permeability) and to retinal neovascularization (by favoring angiogenesis) [14]. Therefore, DR is a clear example of glucose damage to the vascular bed and tissues: Capillary abnormalities in the retina are one of the first pathological changes due to hyperglycemia and correlate with vascular damage in other organs and tissues [17].

Several studies have demonstrated that DR is strongly linked to CV disease. In a systematic review and meta-analysis of observational studies, any degree of DR was significantly correlated with a two-fourfold increased risk for all-cause mortality and/or CV events in both type 1 and type 2 diabetes, compared to diabetic subjects without retinopathy [18]. In the large population study by Schramm et al., all patients with diabetes and on glucose-lowering treatment were found to be at risk of $\mathrm{CV}$ mortality and morbidity, which was similar to that of non-diabetics with prior MI (Fig. 1) [4]. Additionally, the study by Jernberg et al. [19] highlighted that in patients with prior MI, diabetes was an independent risk factor for subsequent ischemic events and death (Fig. 2).

In another trial, CV risk was higher in proliferative than in non-proliferative retinopathy $(18.7 \pm 10.0 \%$ vs. $11.3 \pm 8.4 \%$, $p=0.01$ ), but proliferative retinopathy was also more frequent in a high CV risk group versus a low-risk group (20\% vs. $4.1 \%$, respectively; $p=0.04$ ) [20]. More recently, in diabetic patients with retinopathy, an increased risk ratio (RR) of 2.33 (95\% CI 1.92-2.81) was confirmed for allcause mortality, as well as for stroke $(\mathrm{RR}=1.74,95 \% \mathrm{CI}$ : 1.35-2.24) and heart failure (RR 2.24, 95\% CI 0.98-5.14, $p=0.056)$ [21].

Based on this evidence, it could reasonably be hypothesized that the microvascular alterations observed in the 

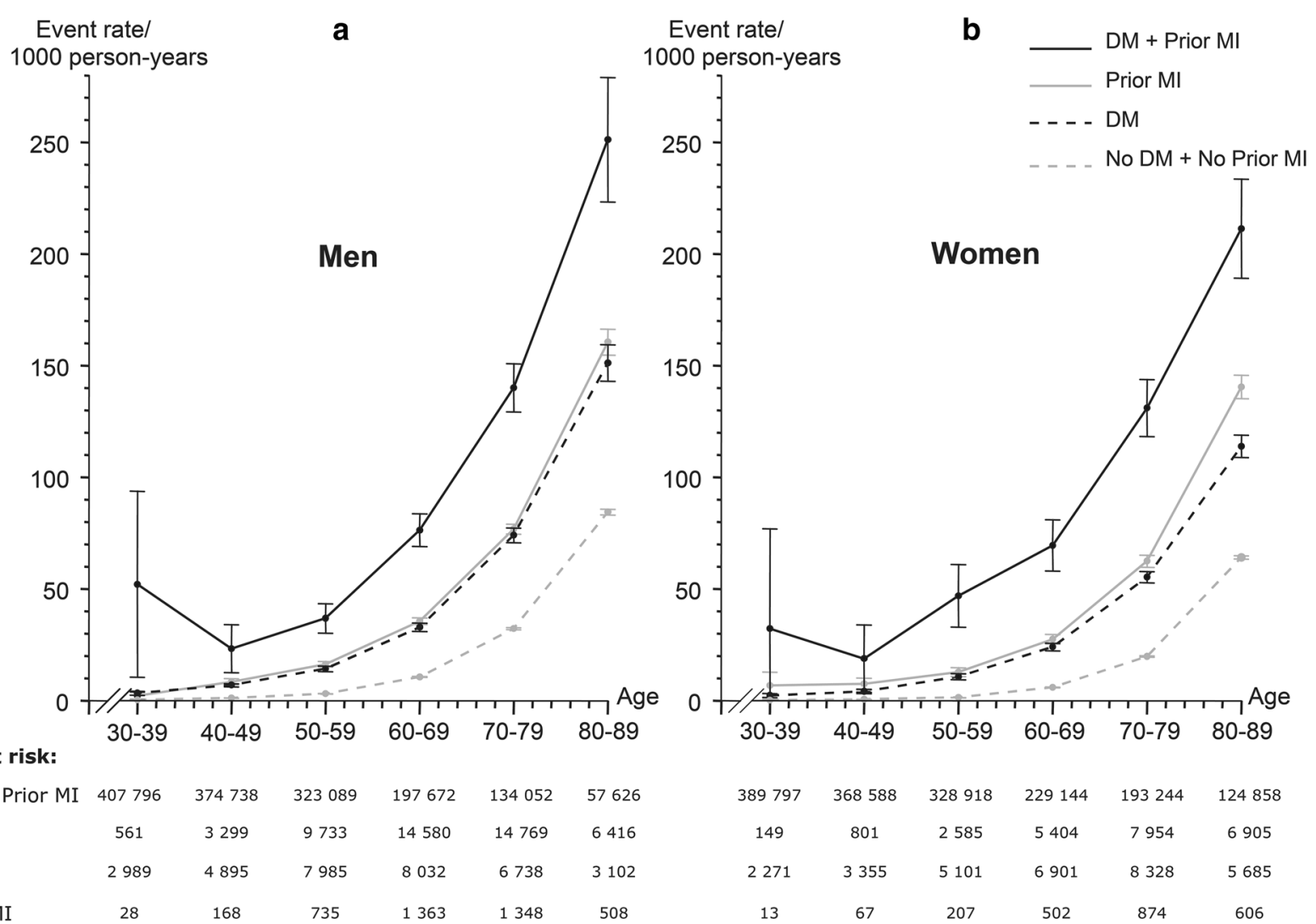

Numbers at risk

$\begin{array}{lcccccc}\text { No DM + No Prior MI } & 407796 & 374738 & 323089 & 197672 & 134052 & 57626 \\ \text { Prior MI } & 561 & 3299 & 9733 & 14580 & 14769 & 6416 \\ \text { DM } & 2989 & 4895 & 7985 & 8032 & 6738 & 3102 \\ \text { DM + Prior MI } & 28 & 168 & 735 & 1363 & 1348 & 508\end{array}$

606

Fig. 1 Event rates for cardiovascular mortality in men (a) and women (b) by age and sex considering diabetes mellitus (DM) and prior myocardial infarction (MI). Modified from Schramm et al. [4]

retina might parallel the pathological changes seen in other organs, such as the heart and brain [21], and might contribute to being able to better define individual CV risk profiles.

\section{Relevant points}

- DR can be considered a clear and easily detectable example of glycemia-induced vascular damage in diabetes, which could also reflect what happens in the CV system.

- Both inflammation and VEGF-mediated pathways play a fundamental role in the pathogenesis of DR and DME.

- DR severity and its deterioration over time are correlated with a progressively increased risk for $\mathrm{CV}$ events compared to patients with diabetes and no retinopathy in both observational studies and randomized clinical trials.

\section{Therapeutic approaches to diabetic macular edema}

Recent decades have witnessed an important evolution in the management of DME. Importantly, metabolic control of glycemia and management of other risk factors (high blood pressure, hyperlipidemia) still remain at the core of preventing the development and progression of the disease $[10,16]$. Laser photocoagulation is no longer the standard of care in DME and is now generally limited to specific forms of the disease, such as the vasogenic subforms or in eyes with a central retinal thickness $(\mathrm{CRT})<300 \mu \mathrm{m}$ or vitreomacular adhesion [16]. Vitreoretinal surgery is a second-line treatment to be used only in specific conditions characterized by vitreous hemorrhage or retinal detachment $[14,16]$. Today, intravitreal pharmacological treatment using antiVEGF agents or corticosteroids is the standard of care for DME [16].

\section{Anti-vascular endothelial growth factor agents}

The development of anti-VEGF agents targets one of the main pathogenetic mechanisms of DME. These molecules inhibit VEGF-A to decrease vascular permeability and to slow the breakdown of the blood-retinal barrier by impeding angiogenesis through a mitogenic effect on endothelial cells. They also enhance both the migration of endothelial cells and adhesion of leukocytes to vascular walls via stimulation of ICAM-1 and VCAM-1 [14]. At present, three different anti-VEGF molecules are commercially available. 
a

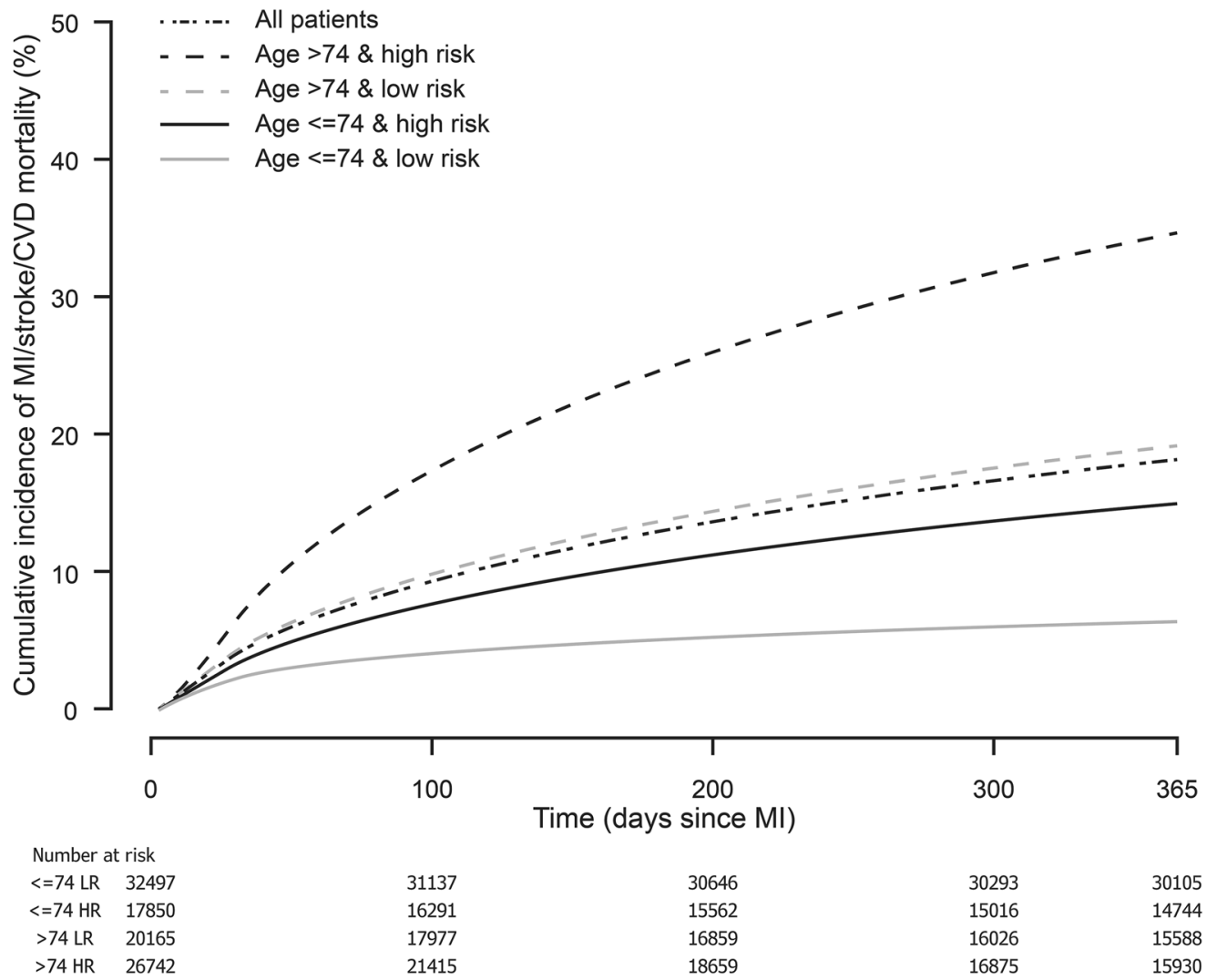

b

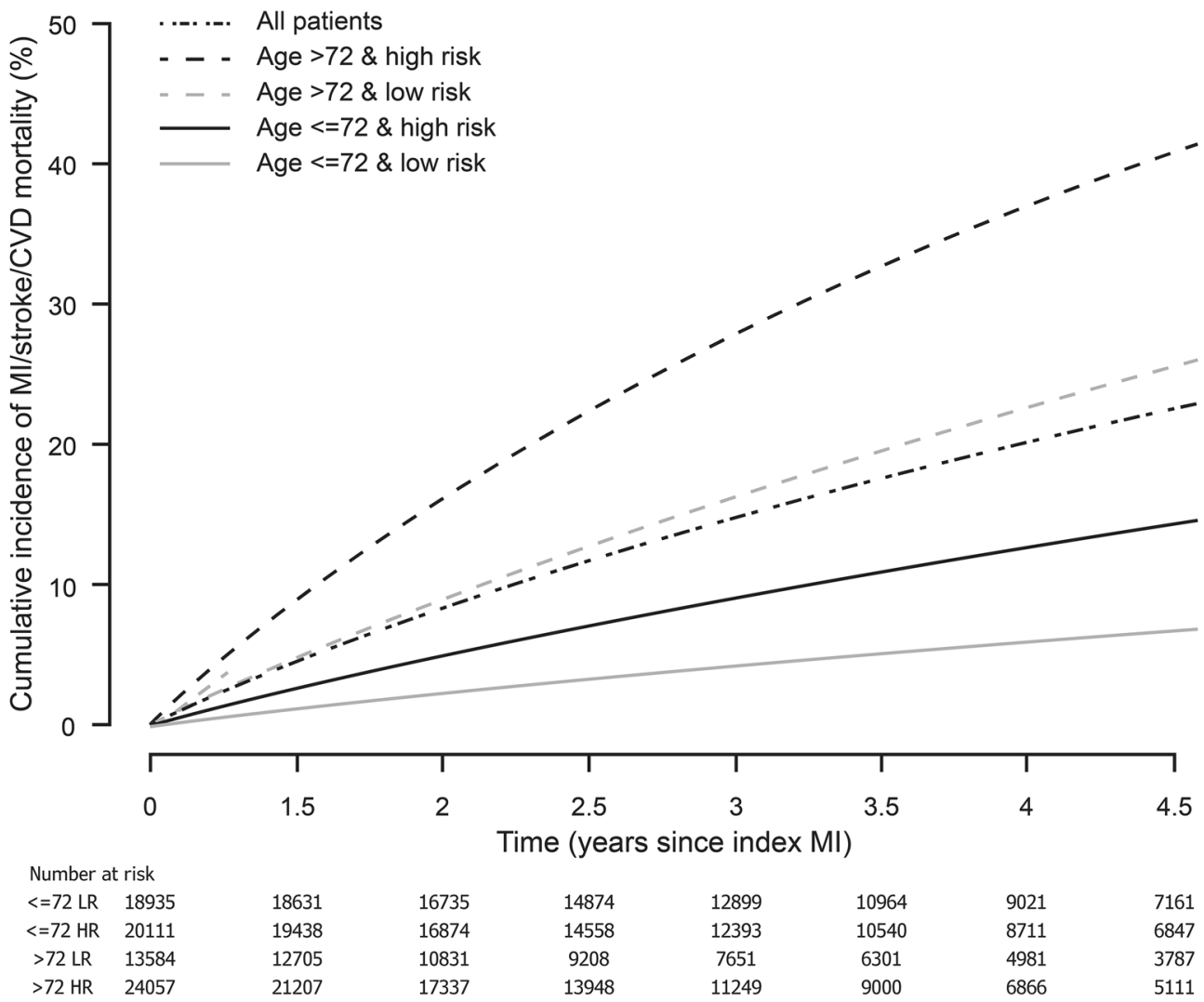


४Fig. 2 Kaplan-Meier estimate for risk of a combined endpoint [myocardial infarction (MI), ischemic stroke, or cardiovascular death] during the first year after the index MI for the entire population (a) and in the subset of stable post-myocardial infarction patients (b) by age and high versus low risk. Modified from Jernberg et al. [19]

Bevacizumab is an anti-VEGF humanized monoclonal antibody, inhibiting the binding of all VEGF isoforms to its receptors. It was first developed for colorectal cancer and was later used as intravitreal therapy for DME [16, 22]. When compared to ranibizumab and aflibercept in the National Institutes of Health (NIH)-sponsored DRCR.net (Diabetic Retinopathy Clinical Research Network) ProtocolT study on patients with DME, it improved the mean bestcorrected visual acuity (BCVA) score after 1 and 2 years, even if to a lower extent than aflibercept in the subpopulation with poorer visual acuity at baseline $(p<0.001$ and $p=0.02$ for aflibercept vs. bevacizumab, at 1 and 2 years, respectively) [23, 24].

Ranibizumab is a humanized monoclonal antibody Fab fragment that was developed for intravitreal use against all isoforms of VEGF-A, inhibiting the binding of VEGF-A to its receptors [16, 25]. In the pivotal RISE and RIDE studies (two parallel, double-masked, twin trials), two different ranibizumab dosages $(0.5 \mathrm{mg}$ and $0.3 \mathrm{mg}$ once a month) were compared to sham injection in 759 subjects with DME $[26,27]$. After 2 years, both ranibizumab doses demonstrated a significantly greater improvement in visual acuity $(p<0.001)$, with a lower number of macular laser procedures $(p<0.0001)$ [26]. The results observed at 24 months were also maintained during the 3-year extension study [27].

Aflibercept is a recombinant fusion protein in which the extracellular domains of human VEGF receptors 1 and 2 are linked to the $\mathrm{Fc}$ fragment of human immunoglobulin (Ig) G1. It acts as a decoy receptor, linking VEGF-A and placental growth factor to reduce their interaction with VEGF receptors [28]. In randomized trials in patients with DME, both ranibizumab and aflibercept showed an improvement in visual acuity versus sham injections and laser therapy [26, 27, 29-31]. The three agents were directly compared in the NIH-sponsored DRCR.net Protocol-T trial, where aflibercept was associated with greater improvement in visual acuity at 1 year compared to bevacizumab and ranibizumab in the subgroup of patients with poorer baseline visual acuity, although at 2 years the difference was significant only compared to bevacizumab [23, 24].

Despite their efficacy, one crucial aspect in the clinical use of anti-VEGF agents is their pharmacokinetics, i.e., intravitreal/systemic half-life and systemic exposure after intravitreal administration, due to a possible impact on the systemic safety profile. Bevacizumab has an intravitreal halflife of 3-6.7 days after a single intravitreous administration [16] and a systemic half-life of 20 days after intravenous infusion [22]. On the other hand, after intravitreous injection, the intravitreal half-life of ranibizumab is 9 days with a systemic half-life of around $2 \mathrm{~h}$ [25, 32]. Less pharmacokinetic data are available for aflibercept, whose systemic half-life after intravenous administration of $2-4 \mathrm{mg} / \mathrm{kg}$ doses is 5-6 days [33].

A recent prospective, non-randomized clinical trial in 151 patients affected by different eye diseases [neovascular age-related macular degeneration (AMD), DME, or retinal vein occlusion (RVO)] demonstrated that after three monthly intravitreal injections of aflibercept $2 \mathrm{mg}$, bevacizumab $1.25 \mathrm{mg}$, or ranibizumab $0.5 / 0.3 \mathrm{mg}$, the systemic concentrations of the anti-VEGF agent were highest with bevacizumab, followed by aflibercept, and lowest with ranibizumab (Fig. 3), probably due to the presence of a $\mathrm{Fc}$ fragment in both bevacizumab and aflibercept, which could be responsible for the reduced clearance through the FcRn-mediated mechanism (which stimulates recycling in the endosomes of endothelial cells). Furthermore, as a direct consequence of increased systemic exposure, among the three molecules, aflibercept produced the greatest inhibition of plasma-free VEGF versus baseline (Fig. 4) [34]. Regardless of differences between individual molecules, it should be stressed that all the available anti-VEGF agents have shown some degree of systemic absorption and, more importantly, inhibition of plasma-free VEGF.

To date, there is no clear picture of the clinical consequences of the systemic VEGF suppression with intravitreal anti-VEGF treatment. It is well known that systemic exposure with intravenous use in oncology is characterized by a wide spectrum of side effects, such as hypertension, arterial thromboembolic accidents, renal dysfunction, gastrointestinal perforations, and compromised wound healing and tissue repair (Fig. 5) [34, 35]. However, the registrative trials in DME used much smaller doses for intravitreal therapy compared to the high intravenous dosages used in oncology, with a generally safe profile of anti-VEGF agents in the overall population [36]. Nevertheless, it should be noted that subjects with an already high level of baseline risk for adverse events from these drugs were usually excluded from clinical studies: In the RISE/RIDE trials, uncontrolled hypertension or diabetes and recent history (within 3 months) of cerebrovascular accident or MI were exclusion criteria; similarly, in the VIVID/VISTA trials, patients with cerebral vascular accident and/or MI within 180 days prior to day 1 were excluded $[26,30,36]$.

The use of the three anti-VEGF agents in patients with DME was directly compared in the Protocol-T study. At 1 year, no differences were seen in the occurrence of serious adverse events, deaths, hospitalization, or CV events [23]. At 2 years, however, ranibizumab showed a higher incidence (12\%) of Antiplatelet Trialists' Collaboration (APTC) events (mainly non-fatal stroke and vascular deaths), compared 

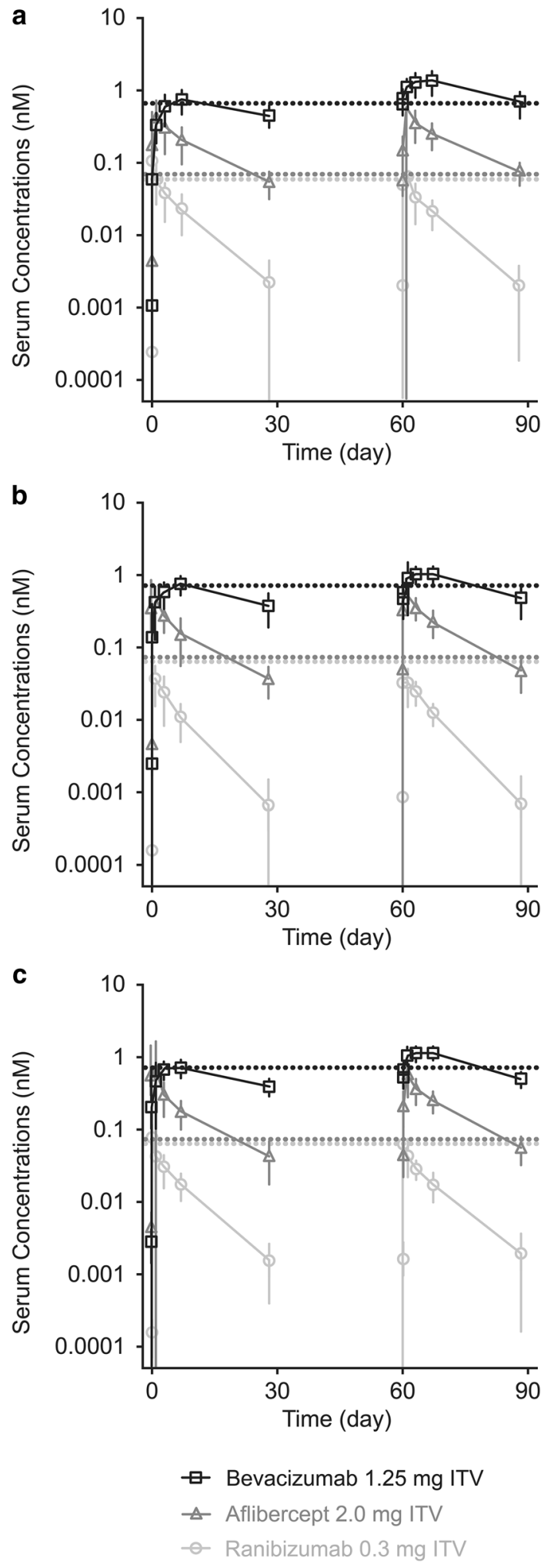

Fig. 3 Mean (SD) serum concentration-time profile for aflibercept, bevacizumab, and ranibizumab after intravitreal administration in (a) patients with age-related macular degeneration (AMD), b patients with diabetic macular edema (DME), and $\mathbf{c}$ patients with retinal vein occlusion (RVO). ITV intravitreal. Modified from Avery et al. [34]

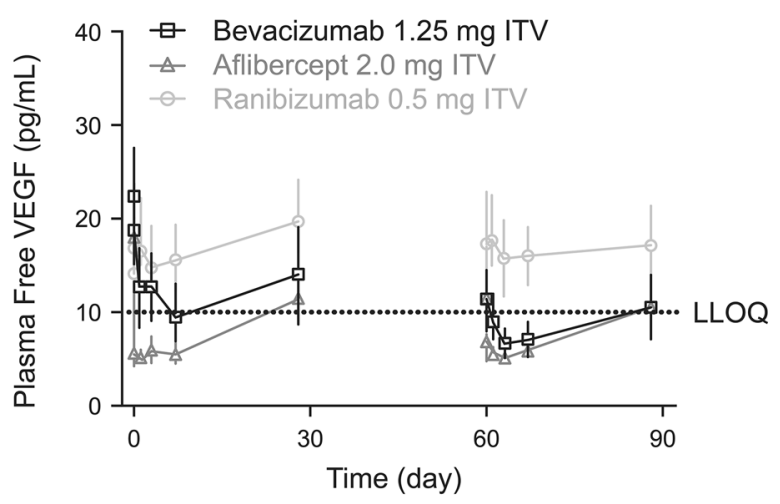

b
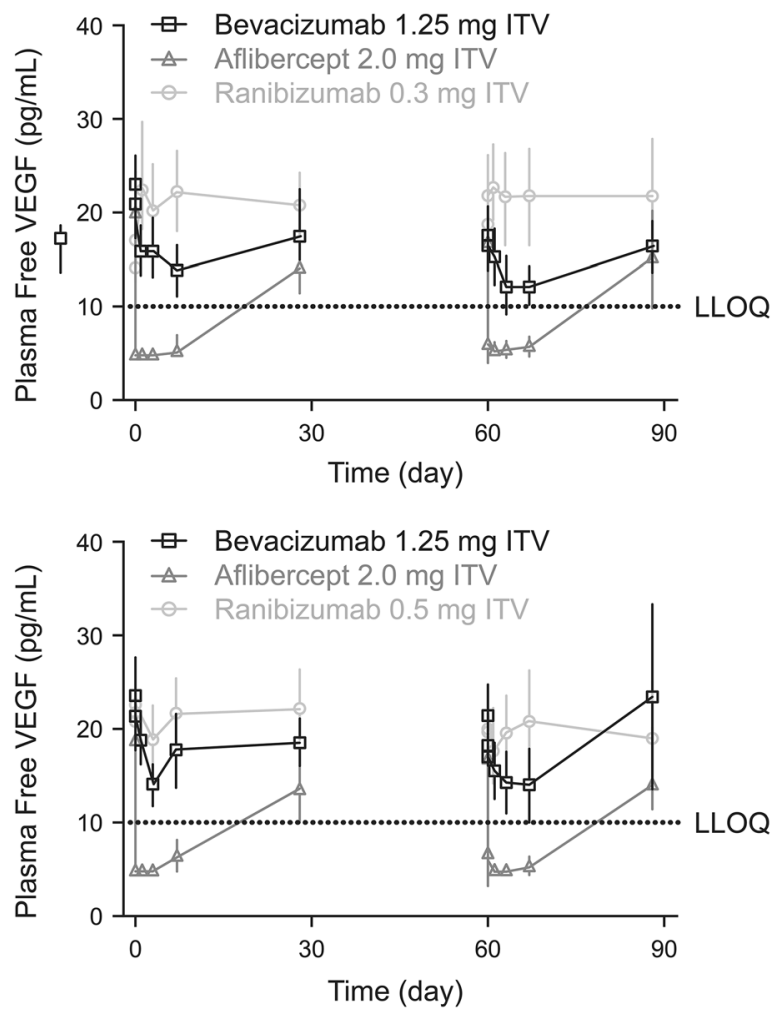

Fig. 4 Mean (95\% CI) plasma-free vascular endothelial growth factor (VEGF) with aflibercept, bevacizumab, and ranibizumab in (a) patients with age-related macular degeneration (AMD), b patients with diabetic macular edema (DME), and $\mathbf{c}$ patients with retinal venin occlusion (RVO). $L L O Q$ lower limits of quantitation; ITV intravitreal. Modified from Avery et al. [34]

to $8 \%$ with bevacizumab and $5 \%$ with aflibercept (global $p=0.047$; pairwise comparisons: $p=0.34$ for aflibercept vs. bevacizumab, $p=0.047$ for aflibercept vs. ranibizumab, and $p=0.20$ for ranibizumab vs. bevacizumab) [24]. More recently, a Cochrane systematic review and network metaanalysis did not show any difference among the three antiVEGF drugs in serious systemic adverse events (i.e., hospitalization, disability, or death) with a moderate-to-high level of evidence. Nevertheless, when trying to exclude 


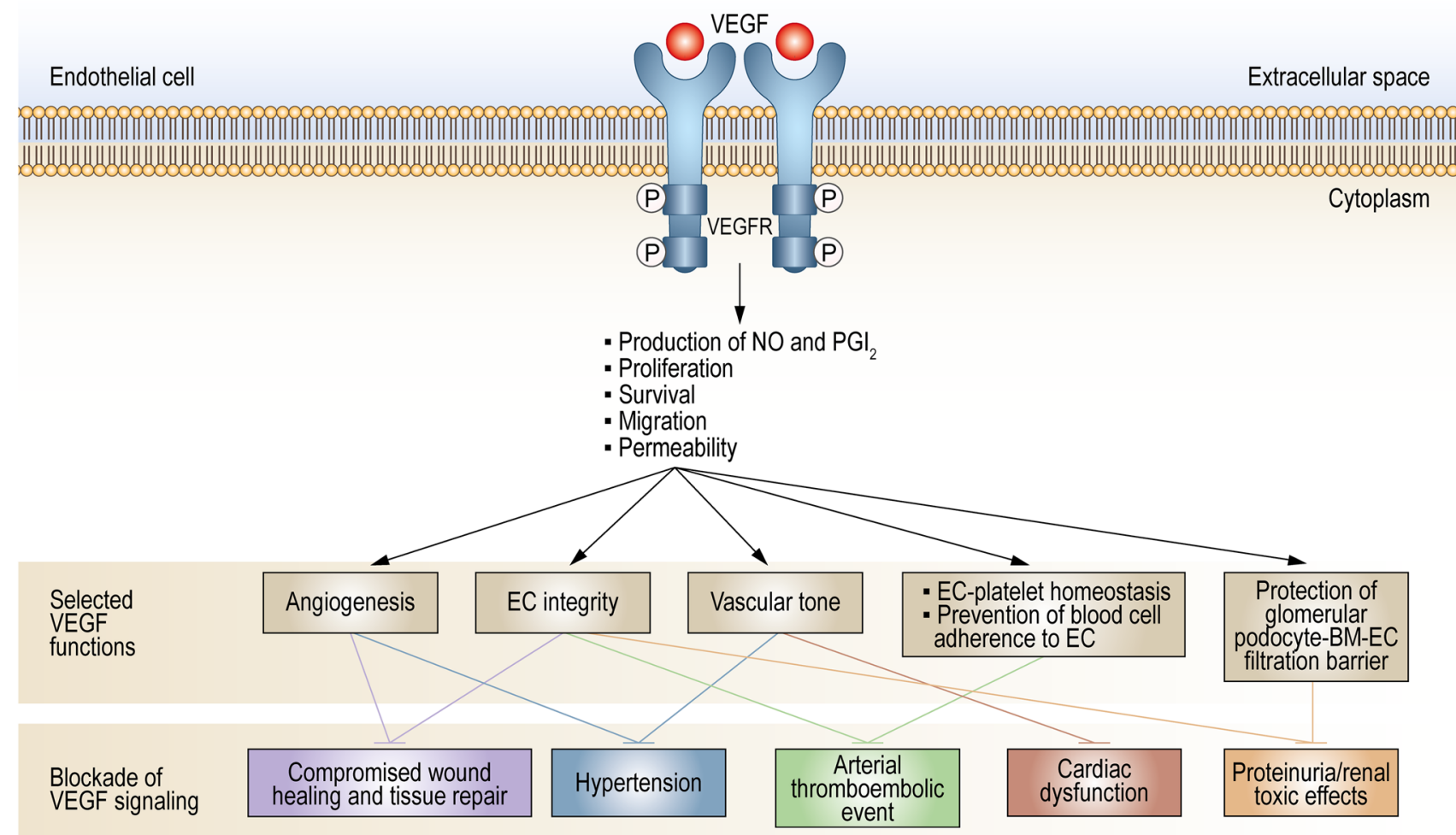

Fig. 5 Physiological functions of VEGF/VEGFR signaling and consequence of pathway blockade. $B M$ basement membrane; $E C$ endothelial cells; $P$ phosphorylated residues; $P G I_{2}$ prostaglandin $\mathrm{I}_{2}$;

differences for less common events such as arterial thromboembolic events (i.e., vascular death, stroke, MI) and death from any cause, the level of evidence was low or very low due to bias, short duration of follow-up, or lack of precision for estimates [37].

Additional information on these agents comes from the RISE and RIDE trials, where the 3-year data showed a higher incidence of serious adverse events that was potentially related to systemic VEGF inhibition at the higher ranibizumab dose $(0.5 \mathrm{mg})$ compared to the lower dose $(0.3 \mathrm{mg})$ and to sham (19.7\% vs. $16.8 \%$ and $13.1 \%$, respectively; statistical comparison was not performed because ranibizumab $0.5 \mathrm{mg}$ was initiated in the sham arm during the third year) [27]. In a post hoc analysis of the Protocol-T study, where patients were grouped based on the presence or absence of a previous history of stroke or MI at baseline, the incidence of APTC adverse events was higher in the subgroup at major CV risk $(10 \%, 20 \%$, and $36 \%$ with aflibercept, bevacizumab, and ranibizumab, respectively) compared to the subgroup at lower risk (5\%,6\%, and 9\%, respectively), stressing the importance of baseline CV status for the systemic safety profile of anti-VEGF agents (Table 1) [24]. The finding was further confirmed in a recent systematic review and metaanalysis evaluating the systemic safety of aflibercept and
$N O$ nitric oxide; $V E G F$ vascular endothelial growth factor; VEGFR vascular endothelial growth factor receptor. Reproduced from Chen et al. [35]

ranibizumab in subjects considered at high risk for arteriothrombotic events, such as those with diabetes and DME, who were intensively exposed to the drugs for 2 years with monthly injections. An increased risk of death was demonstrated in the anti-VEGF arm (combined aflibercept and $0.5 \mathrm{mg}$ ranibizumab) compared to sham/laser group [odds ratio (OR) 2.98; 95\% CI, 1.44-6.14; $p=0.003$ ], together with an increase in vascular-related death (OR, 2.51; $95 \%$ CI, 1.08-5.82; $p=0.03$ ) and cerebrovascular accidents (OR, $2.33 ; 95 \%$ CI, $1.04-5.22 ; p=0.04)$ [36].

Pregnancy represents another situation in which management of DME could be challenging, because of concerns of the potential deleterious antiangiogenic effects of antiVEGF drugs on a developing fetus. For the intravitreal injection of aflibercept, the FDA advises women of reproductive potential to use contraception before the initial dose, during treatment, and for at least 3 months after the last administration. Nevertheless, although cases of early fetal decease have been reported, most cases in the literature have not shown any demonstrable damage related by intravitreal anti-VEGF therapy during pregnancy. As diabetes and systemic comorbidities in diabetic patients are known risk factors for miscarriage or pregnancy complications, a cause-effect relationship has not been firmly established, as 
Table 1 Post hoc analysis from Protocol-T of APTC adverse events stratified by prior myocardial infarction/stroke. Modified from Wells et al. [24]

\begin{tabular}{|c|c|c|c|}
\hline & Aflibercept $(n=203)$ & Bevacizumab $(n=193)$ & $\begin{array}{l}\text { Ranibi- } \\
\text { zumab } \\
(n=193)\end{array}$ \\
\hline \multicolumn{4}{|c|}{ Participants with no myocardial infarction or stroke prior to baseline } \\
\hline \multicolumn{4}{|c|}{ Vascular events according to the Antiplatelet Trialists' Collaboration occurring at least once at any time during the study, $n$ (\%) } \\
\hline Non-fatal myocardial infarction & $6(3)$ & $3(2)$ & $4(2)$ \\
\hline Non-fatal stroke & $2(<1)$ & $5(3)$ & $6(3)$ \\
\hline Vascular death $^{\mathrm{a}}$ & $2(<1)$ & $4(2)$ & $7(4)$ \\
\hline \multirow[t]{2}{*}{ Any Antiplatelet Trialists' Collaboration event } & $10(5)$ & $12(6)$ & $17(9)$ \\
\hline & Aflibercept $(n=21)$ & Bevacizumab $(n=25)$ & $\begin{array}{l}\text { Ranibi- } \\
\text { zumab } \\
(n=25)\end{array}$ \\
\hline \multicolumn{4}{|c|}{ Participants with myocardial infarction or stroke prior to baseline } \\
\hline \multicolumn{4}{|c|}{ Vascular events according to the Antiplatelet Trialists' Collaboration occurring at least once at any time during the study, $n(\%)$} \\
\hline Non-fatal myocardial infarction & $1(5)$ & $0(0)$ & $2(8)$ \\
\hline Non-fatal stroke & $0(0)$ & $1(4)$ & $5(20)$ \\
\hline Vascular death $^{\mathrm{a}}$ & $1(5)$ & $4(16)$ & $2(8)$ \\
\hline Any Antiplatelet Trialists' Collaboration event & $2(10)$ & $5(20)$ & $9(36)$ \\
\hline
\end{tabular}

APTC Antiplatelet Trialists' Collaboration [66]

Global $p$ value adjusting for prior myocardial infarction, prior stroke, $p=0.06$

${ }^{a}$ From any potential vascular or unknown cause

recently stated by Peracha and Rosenfeld [38]. The current shared practice is postponing anti-VEGF therapy in pregnant or breast-feeding patients, because of presumed risk to the child. Pre-menopausal women requiring anti-VEGF treatment should undergo pregnancy test to exclude childbearing status, and contraception should be used during therapy. Alternative therapies for DME should be also considered, as intravitreal corticosteroids or focal laser [39].

As a consequence, although intravitreal anti-VEGF agents seem to be generally safe, attention should be given to patients who are at higher risk for $\mathrm{CV}$ disease, such as those with diabetes and those with previous/recent myocardial or cerebrovascular accidents, and in pre-menopausal women, as the sustained suppression of systemic VEGF could be related to potential safety issues $[16,36]$. The fact that there are limited data on the systemic safety of anti-VEGFs in different patient subgroups is also mentioned in the respective Summaries of Product Characteristics, and caution is suggested in treating these patient populations [25, 28]. Accurate evaluation of the CV risk profile of patients with DME is thus warranted in order to appropriately use an anti-VEGF agent in the right patient at the right time. Furthermore, considering the long-term persistence of increased $\mathrm{CV}$ risk in such subgroups of patients [19] and the need for repeated treatment, rigorous evaluation should be maintained even in the long term.

\section{Relevant points}

- Anti-VEGF agents are an important option in the therapeutic armamentarium for patients with DME, which can improve visual acuity and reduce loss of vision.

- The pharmacokinetic characteristics (intravitreal/systemic half-life and systemic exposure after intravitreal administration) are different for the currently available anti-VEGFs: Systemic concentrations are highest with bevacizumab, while aflibercept is responsible for the greatest inhibition of plasma-free VEGF even if all agents show some degree of inhibition.

- Systemic exposure to intravenous anti-VEGFs in oncology is linked to a clear pattern of side effects, and the medical community is trying to better understand their systemic safety profile after intravitreal administration.

- While intravitreous anti-VEGFs seem to be generally safe in the overall population of patients with DME, a safety signal for death and vascular accidents has been shown in subjects who are at risk for $\mathrm{CV}$ disease at baseline and who are intensively treated with monthly injections.

- Moreover, anti-VEGF should not be offered in pregnant women and contraception should be advised during treatment.

- As a consequence, baseline CV risk and pregnancy status should be carefully assessed in every patient 
with diabetes in order to appropriately administer antiVEGF agents; patients with a prior CV event in the previous 3 months, DR, and/or DME are not candidates for anti-VEGF therapy as they were excluded from major registrative trials and there is limited safety information available.

- Considering that $\mathrm{CV}$ risk persists in the long term, a similarly comprehensive approach should be maintained in the long term.

\section{Corticosteroids}

Inflammation is another crucial player in the pathogenesis of DR. The hyperglycemia-induced pathways related to DME include those involved in the generation of AGEs and reactive oxidative species, to the synthesis of proinflammatory agents, such as adhesion molecules, cytokines, and VEGF, as well as protein kinase C [40]. The final outcome is the disruption of the blood-retinal barrier, due to damage to endothelial cells, alterations in tight junction proteins, and increased permeability $[16,41]$. Corticosteroids, due to their anti-inflammatory action, are able to counteract these effects, reducing migration of leukocytes and suppressing the production of cytokines and interleukins, including VEGF [16, 42].

At present, three corticosteroids are commercially available for intravitreal use: triamcinolone acetonide, dexamethasone, and fluocinolone acetonide. The structural variations between the three molecules lead to differences in receptor binding, solubility, and pharmacokinetics [43]. Dexamethasone and fluocinolone acetonide have similar anti-inflammatory potency (higher than triamcinolone) [43], while dexamethasone shows a twofold higher hydrosolubility than fluocinolone, with a consequently greater receptor binding affinity and bioavailability [43]. Higher aqueous solubility also means that its half-life is reduced in the vitreous, which is why dexamethasone is used in a prolonged-release biodegradable implant that maintains a sustained drug concentration in the vitreous over time (6 months) [42, 43].

From a pharmacokinetic standpoint, most of the data on the three molecules are from animal studies, with few investigations in humans. In a study on monkey eyes receiving bilateral $0.7 \mathrm{mg}$ dexamethasone implants (DEX-I), drug delivery followed a bi-phasic time course in the retina and vitreous, with higher intravitreal concentrations in the first 2 months followed by lower levels for up to 6 months. The evaluation of the biological activity of dexamethasone through the expression of CYP3A8 demonstrated increased levels in the retina for 6 months [44]. No data in humans are available for dexamethasone. Regarding fluocinolone acetonide implants, a study performed in rabbits demonstrated a concentration peak in the vitreous at 2 days after administration and a progressive decline during the first 3 months [43]. In a clinical trial in patients with DME, intravitreal insertion at a dose of 0.2- or 0.5-micrograms/day led to peaks at 1 week and at 1 month after administration, which slowly declined thereafter, although therapeutic concentrations were maintained for up to 1 year [45]. Among the three available corticosteroids, triamcinolone acetonide has the shortest half-life in the aqueous humor after intravitreous administration, ranging from 3.2 days in vitrectomized to 18.6 days in non-vitrectomized human eyes [43].

Water solubility also has an important impact on steroid binding to the trabecular meshwork and lens, which increases with increasing lipophilicity, and which may be responsible for some class side effects, such as cataract and increased intraocular pressure (IOP). The latter is directly related to steroid partitioning in the above-mentioned tissues, with a consequent increase in intraocular volume or obstruction of trabecular meshwork due to precipitation (such as for triamcinolone acetonide) [46, 47]. Dexamethasone is characterized by the highest aqueous solubility and lowest lipophilicity compared to triamcinolone acetonide and fluocinolone acetonide, leading to a very low level of binding to the trabecular meshwork and lens in bovine/human ocular tissues [47]. This aspect may be responsible for a lower incidence of increased IOP and cataract compared to the other two molecules.

A systematic review and meta-analysis of prospective randomized studies, prospective cohort trials, and retrospective studies demonstrated an incidence of ocular hypertension (OHT) of $32 \%$ in subjects treated with $4 \mathrm{mg}$ triamcinolone acetonide, 66-79\% with fluocinolone acetonide implants $(0.59 \mathrm{mg}$ and $2.1 \mathrm{mg}$ doses, respectively), and $11-15 \%$ with dexamethasone implants (0.35 and $0.7 \mathrm{mg}$ doses, respectively) [46]. In the MEAD registrative trials (two randomized, sham-controlled, phase 3 identical protocols), 3-year data showed an increase in IOP from baseline in $30.8 \%$ of DME patients treated with DEX-I $0.7 \mathrm{mg}$ (the approved dose), requiring surgical or laser in only $1.2 \%$ of subjects and usually managed with IOP-lowering drugs $[41,48]$. There was no cumulative effect on IOP from repeated dexamethasone implants since no increase in IOP-related events was seen over time and the proportion of patients using IOP-lowering drugs during the 3-year observation remained stable [41]. Cataractrelated adverse events were observed in $67.9 \%$ of subjects, and cataract surgery was needed in $59.2 \%$ of cases [41]. In the FAME studies (two parallel, randomized, phase 3 protocols in patients with DME), $0.2 \mu \mathrm{g} /$ day fluocinolone acetonide showed, after 3 years, increased IOP in $37.1 \%$ of patients (needing trabeculoplasty in $1.3 \%$ and incisional glaucoma surgery in $4.8 \%$ of cases) and occurrence of cataract in $81.7 \%$ of treated subjects (in the high dose of 
$0.5 \mu \mathrm{g} / \mathrm{day}$; increased IOP and cataract were observed in $88.7 \%$ and $45.5 \%$ of enrolled subjects, respectively) [49].

Based on these characteristics and differences, Guidelines for the Management of Diabetic Macular Edema from the European Society of Retina Specialists (EURETINA) recommend dexamethasone as first choice among corticosteroids, followed by fluocinolone in case of nonsteroid responders with chronic macular edema that does not respond to other therapies, while triamcinolone, characterized by a poorer safety profile on IOP and cataract and the absence of a specific indication for DME, should be used only in patients who are not candidates for other drugs [16].

Dexamethasone is provided in an intravitreal, rod-shaped biodegradable implant containing 700 micrograms of active drug, which is slowly released for up to 6 months, thus circumventing the need for monthly injections [42]. Regulatory approval for the $0.7 \mathrm{mg}$ implant for clinical use in DME was based on several clinical trials performed in patients with diabetes where the implant, in association with laser therapy or as monotherapy, was compared to laser therapy alone or to sham procedure. In the above-mentioned MEAD trials on a total of 1048 DME patients, the proportion of patients reaching improvement in $\mathrm{BCVA} \geq 15$ letters from baseline to the end of the study was significantly higher in the $0.7 \mathrm{mg}(22.2 \%)$ and $0.35 \mathrm{mg}$ dexamethasone implant groups $(18.4 \%)$ versus the sham arm $(12 \% ; p \leq 0.0018$ for both comparisons) after a mean number of injections of 4.1 , 4.4 , and 3.3, respectively, over 3 years. This clinical efficacy was coupled with a significant reduction in CRT from baseline, and the improvement in visual acuity was independent of lens status at baseline and observed in both phakic and pseudophakic eyes [41].

One important aspect seen during the clinical development of the dexamethasone implant is related to its systemic safety profile. It was shown that intravitreal administration of dexamethasone provided optimal concentrations to the vitreous without systemic effects: In particular, in a preclinical study on rabbits, the pharmacokinetic systemic profile of dexamethasone phosphate and its metabolite dexamethasone, at low $(25 \mu \mathrm{g} / \mathrm{kg})$ and high $(250 \mu \mathrm{g} / \mathrm{kg})$ doses, was explored through different routes of administration to the retina (intravenous, subconjunctival, and intravitreal) [50]. Plasma concentrations were higher, and plasma elimination was fastest after intravenous administration, followed by a subconjunctival route; intravitreal administration was characterized by the lowest plasma concentrations during 24-h monitoring and by a depot effect, which make it suitable for drug delivery in the back of the eye [50]. Several studies in humans exploring, through different study designs, topical, oral, peribulbar, subconjunctival, and intravitreal dexamethasone administration, helped to characterize the local intravitreous and systemic pharmacokinetic profile of each route, highlighting that the intravitreal use of dexamethasone achieves the highest local concentrations with the lowest systemic distribution [51-55].

These pharmacokinetic characteristics influence the systemic safety profile of dexamethasone. The 3-year results from MEAD trials in patients with DME showed no difference between the dexamethasone arms and sham in systemic serious adverse events, which included cardiac disorders (such as coronary artery disease and MI), cerebrovascular accidents, and vascular events [41]. This finding was confirmed in a recent systematic review of real-world studies performed with repeated use of a $0.7 \mathrm{mg}$ dexamethasone implant in subjects affected by DME: A good overall safety profile was seen in the long term (mean follow-up duration ranged from 5.5 to 23 months in the different studies), even in the presence of a shorter retreatment time than usually indicated (5.3 vs. 6 months) [56]. Data from real-world experiences in DME are, indeed, an important piece of information on long-term dexamethasone use, considering the chronic nature of this therapy in a population with persistently high CV risk over time. One of the largest real-life studies assessing the safety of intravitreal dexamethasone implant injections in various retinal conditions including diabetic macular edema (1434 eyes) has recently confirmed both the local and systemic safety of the implant; cataract progression and intraocular pressure rise were the most common side effects, but they were easily manageable with a limited number of additional procedures [57]. Finally, the systemic safety profile of the dexamethasone implant is also reflected in the Summary of Product Characteristics, where the only reported systemic adverse drug reactions are headache and migraine [42].

Therefore, in taking a holistic approach to the patient with diabetes and macular edema, the favorable efficacy profile and minimal systemic adverse events for intravitreal corticosteroids, especially the dexamethasone implant, may represent a fundamental element in the therapeutic choice when considering the high $\mathrm{CV}$ risk of some of these patients. Indeed, the EURETINA Guidelines, which consider pharmacological intravitreal treatments as a first-line approach to DME, although recognizing the fundamental role of antiVEGF drugs, consider corticosteroids as a crucial component of the therapeutic armamentarium. Thus, steroids, and firstly dexamethasone, must no longer be considered as the second choice, especially when considering the wider effects of VEGF inhibition [16, 58]. Corticosteroids should be used as a first-line therapy in the subset of patients with a history of or recent major $\mathrm{CV}$ events in both the short and long term (even years after the event), considering on the one hand that these patients were excluded from registrative trials with anti-VEGFs and, on the other, the recently demonstrated increased risk for death and cerebrovascular accidents in 
high-risk patients treated with anti-VEGF agents for 2 years $[16,36]$.

\section{Relevant points}

- Use of corticosteroids for the treatment of DME can target the multifaceted inflammatory pathways involved in the pathogenesis of DR, with a more comprehensive spectrum of action compared to anti-VEGF agents (which act only on one component of the inflammatory cascade).

- The steroids (triamcinolone acetonide, fluocinolone acetonide, and dexamethasone) used in DME are not all the same, and differences in structure, anti-inflammatory potency, pharmacokinetics, receptor binding, water solubility, and effect on the trabecular meshwork can have an impact on the efficacy and safety profiles.

- Dexamethasone shows the highest aqueous solubility and, as a consequence, a short half-life that necessitates administration with a prolonged-release intravitreal implant. This characteristic is also responsible for the very low level of binding to the trabecular meshwork and lens. From a clinical standpoint, this aspect is strictly related to the lower incidence of ocular side effects (cataract and increased IOP) observed with dexamethasone as compared to the other two steroids in clinical trials.

- An intravitreal route of administration has been identified as the best way to provide higher local intravitreal dexamethasone concentrations with low levels of systemic exposure. This characteristic means that the incidence of serious systemic adverse events (including cardiac disorders and vascular events) is similar between active dexamethasone treatment arms and sham/laser therapy in clinical trials, with a good overall safety profile in realworld studies.

- The favorable systemic safety profile, even in the long term, clearly differentiates corticosteroids from antiVEGF agents since these latter agents may inhibit plasma-free VEGF due to their systemic presence.

- EURETINA Guidelines suggest that corticosteroids, and dexamethasone as the preferred agent, should be considered as first-line therapy in patients with high $\mathrm{CV}$ risk, such as a history of or recent major $\mathrm{CV}$ events.

\section{Clinical use of steroids in diabetic macular edema}

In addition to patients with high $\mathrm{CV}$ risk, other patient categories might benefit from the clinical use of steroids for DME. Corticosteroids can be considered as a first choice in subjects who are not willing or not compliant with monthly treatment (such as in the case of anti-VEGF agents) or more frequent monitoring visits [16]. In a recent trial comparing dexamethasone intravitreal implant and ranibizumab in DME, steroid use was non-inferior to antiVEGF agents in terms of improvement of visual acuity at 12 months with a similar reduction in CRT, coupled with a lower mean number of injections per patient ( 2.85 vs. 8.70 implants with dexamethasone and ranibizumab, respectively) [59]. Clinical studies have suggested that eyes with DME switched to dexamethasone implant because they were considered refractory to anti-VEGF therapy after three monthly injections had better visual and anatomical outcomes at 12 months than those that continued anti-VEGF treatment [60]. It should be highlighted that the relationship between frequency of administration and adherence to/persistence on treatment is well known, especially in chronic conditions such as diabetes [61, 62], and that intermittent dosing (for example, once-weekly administration) has been associated with an improvement in adherence [63].

Corticosteroids are also a valuable alternative in pseudophakic patients (phakic patients should be informed about the risk of cataract with steroid use), in non-responders to anti-VEGF therapy, and in patients in whom anti-VEGF therapy is contraindicated (such as pregnancy, in addition to high CV risk) [16, 58]. Finally, intravitreal dexamethasone may reduce peripheral retinal ischemia [64], thus improving severity and delaying progression to proliferative DR [65].

\section{Conclusions}

Management of DR is a complex task requiring a multidisciplinary approach. A close relationship between the diabetologist and ophthalmologist is fundamental to simultaneously control for metabolic factors (such as glycemia, hypertension, and hyperlipidemia), assess the CV risk profile, and manage DME with the appropriate treatment in the individual patient. The vascular neurologist should consider the increased risk of stroke in patients with DR, and patients with stroke and a past medical history of diabetes or first diagnosed with diabetes during hospitalization in the stroke unit should undergo evaluation by an ophthalmologist. In patients with DME, concomitant pathologies, presence of multiple diabetic complications, level and long-term persistence of CV risk, as well as patient compliance, should be carefully evaluated, with the aim of establishing a tailored therapy for ocular disease without losing a focus on a holistic approach to diabetes management. In this context, $\mathrm{CV}$ risk assessment is crucial for an appropriate therapeutic choice in DME, with recent guidelines suggesting the use of corticosteroids, and in particular dexamethasone intravitreal implant, as a well-tolerated and efficacious first-line treatment in patients with a history of or recent $\mathrm{CV}$ events. 
In this subset of patients, the use of corticosteroids should be applied with no time restriction considering the first CV event. Real-world experience on the chronic use of dexamethasone supports its long-term safety profile, which is an essential consideration in the light of the need for prolonged treatment and the increased CV risk present in patients with diabetes and DR/DME.

Acknowledgements We thank Ray Hill, an independent medical writer, who provided English language editing and journal styling prior to submission on behalf of Health Publishing \& Services Srl and funded by Allergan SpA, Italy. All authors met the ICMJE authorship criteria. Neither honoraria nor payments were made for authorship.

Author's contribution All authors contributed to the review conception and design of the literature search. All authors contributed to the drafting and critical revision of the manuscript, commented on previous versions of the manuscript, and read and approved the final manuscript prior to submission.

Data availability Data sharing is not applicable to this article as no datasets were generated or analyzed during the current study.

\section{Compliance with ethical standards}

Conflict of interest Francesco Bandello has acted as a consultant for Allergan, Boehringer-Ingelheim, Fidia Sooft, Hoffmann La Roche, Novartis, NTC Pharma, SIFI, Thrombogenics, and Zeiss. Danilo Toni has received honoraria from Bayer, Boehringer Ingelheim, Pfizer, Bristol-Myers Squibb, Daiichi Sankyo, and Medtronic. Massimo Porta has received Advisory Board fees from Novartis, SIFI, and Allergan. Monica Varano has received Advisory Board fees from Novartis, Bayer, SIFI, and Allergan.

Ethical approval This article does not contain any studies with human participants or animals performed by any of the authors.

Informed consent For this type of study, formal consent is not required.

\section{References}

1. International Diabetes Federation (2017) IDF Diabetes Atlas 8th Edition. https://diabetesatlas.org/resources/2017-atlas.html.40-50. Accessed 13 July 2019

2. World Health Organization (WHO) (2016) Global report on diabetes. https://www.who.int/diabetes/global-report/en/. Accessed 13 July 2019

3. Haffner SM, Lehto S, Ronnemaa T, Pyorala K, Laakso M (1998) Mortality from coronary heart disease in subjects with type 2 diabetes and in nondiabetic subjects with and without prior myocardial infarction. N Engl J Med 339(4):229-234. https://doi. org/10.1056/NEJM199807233390404

4. Schramm TK, Gislason GH, Kober L et al (2008) Diabetes patients requiring glucose-lowering therapy and nondiabetics with a prior myocardial infarction carry the same cardiovascular risk: a population study of 3.3 million people. Circulation 117(15):19451954. https://doi.org/10.1161/circulationaha.107.720847

5. World Health Organization (WHO) (2018) Fact sheet: the top 10 causes of death. https://www.who.int/news-room/fact-sheets/detai 1/the-top-10-causes-of-death. Accessed 13 July 2019
6. Brun E, Nelson RG, Bennett PH et al (2000) Diabetes duration and cause-specific mortality in the Verona Diabetes Study. Diabetes Care 23(8):1119-1123. https://doi.org/10.2337/diacare.23.8.1119

7. Chen R, Ovbiagele B, Feng W (2016) Diabetes and stroke: epidemiology, pathophysiology, pharmaceuticals and outcomes. Am J Med Sci 351(4):380-386. https://doi.org/10.1016/j.amjms .2016.01.011

8. Piepoli MF, Hoes AW, Agewall S et al (2016) 2016 European Guidelines on cardiovascular disease prevention in clinical practice: the Sixth Joint Task Force of the European Society of Cardiology and Other Societies on Cardiovascular Disease Prevention in Clinical Practice (constituted by representatives of 10 societies and by invited experts) developed with the special contribution of the European Association for Cardiovascular Prevention \& Rehabilitation (EACPR). Eur Heart J 37(29):2315-2381. https://doi. org/10.1093/eurheartj/ehw106

9. American Diabetes Association (2019) 10. Cardiovascular disease and risk management: standards of medical care in diabetes-2019. Diabetes Care 42(Suppl 1):S103-S123. https://doi. org/10.2337/dc19-s010

10. American Diabetes Association (2019) 11. Microvascular complications and foot care: standards of medical care in diabetes-2019. Diabetes Care 42(Suppl 1):S124-S138. https://doi. org/10.2337/dc19-s011

11. Pearce I, Simó R, Lövestam-Adrian M, Wong DT, Evans M (2019) Association between diabetic eye disease and other complications of diabetes: implications for care. A systematic review. Diabetes Obes Metab 21(3):467-478. https://doi. org/10.1111/dom.13550

12. Lee R, Wong TY, Sabanayagam C (2015) Epidemiology of diabetic retinopathy, diabetic macular edema and related vision loss. Eye Vis (Lond) 2:17. https://doi.org/10.1186/s40662-015-0026-2

13. International Council of Ophthalmology (2017) Updated 2017 guidelines for diabetic eye care. http://www.icoph.org/enhan cing_eyecare/international_clinical_guidelines.html. Accessed 13 July 2019

14. Simo R, Sundstrom JM, Antonetti DA (2014) Ocular anti-VEGF therapy for diabetic retinopathy: the role of VEGF in the pathogenesis of diabetic retinopathy. Diabetes Care 37(4):893-899. https://doi.org/10.2337/dc13-2002

15. Yau JW, Rogers SL, Kawasaki R et al (2012) Global prevalence and major risk factors of diabetic retinopathy. Diabetes Care 35(3):556-564. https://doi.org/10.2337/dc11-1909

16. Schmidt-Erfurth U, Garcia-Arumi J, Bandello F et al (2017) Guidelines for the management of diabetic macular edema by the European Society of Retina Specialists (EURETINA). Ophthalmologica 237(4):185-222. https://doi.org/10.1159/000458539

17. Gerstein HC, Werstuck GH (2013) Dysglycaemia, vasculopenia, and the chronic consequences of diabetes. Lancet Diabetes Endocrinol 1(1):71-78. https://doi.org/10.1016/S2213-8587(13)70025 $-1$

18. Kramer CK, Rodrigues TC, Canani LH, Gross JL, Azevedo MJ (2011) Diabetic retinopathy predicts all-cause mortality and cardiovascular events in both type 1 and 2 diabetes: meta-analysis of observational studies. Diabetes Care 34(5):1238-1244. https:// doi.org/10.2337/dc11-0079

19. Jernberg T, Hasvold P, Henriksson M, Hjelm H, Thuresson M, Janzon M (2015) Cardiovascular risk in post-myocardial infarction patients: nationwide real world data demonstrate the importance of a long-term perspective. Eur Heart J 36(19):1163-1170. https://doi.org/10.1093/eurheartj/ehu505

20. Shoeibi N, Bonakdaran S (2017) Is there any correlation between diabetic retinopathy and risk of cardiovascular disease? Curr Diabetes Rev 13(1):81-86. https://doi.org/10.2174/157339981266615 1012115355 
21. Zhu XR, Zhang YP, Bai L, Zhang XL, Zhou JB, Yang JK (2017) Prediction of risk of diabetic retinopathy for all-cause mortality, stroke and heart failure: evidence from epidemiological observational studies. Medicine (Baltimore) 96(3):e5894. https://doi. org/10.1097/MD.0000000000005894

22. European Medicines Agency (EMA) (2018) Avastin (bevacizumab) Summary of product characteristics. https://www.ema. europa.eu/en/medicines/human/EPAR/avastin\#product-informatio n-section. Accessed 13 July 2019

23. Wells JA, Glassman AR, Ayala AR et al (2015) Aflibercept, bevacizumab, or ranibizumab for diabetic macular edema. N Engl J Med 372(13):1193-1203. https://doi.org/10.1056/NEJMoa1414 264

24. Wells JA, Glassman AR, Ayala AR et al (2016) Aflibercept, bevacizumab, or ranibizumab for diabetic macular edema: two-year results from a comparative effectiveness randomized clinical trial. Ophthalmology 123(6):1351-1359. https://doi.org/10.1016/j. ophtha.2016.02.022

25. European Medicines Agency (EMA) (2018) Lucentis (ranibizumab) summary of product characteristics. https://www.ema. europa.eu/en/medicines/human/EPAR/lucentis\#product-infor mation-section. Accessed 13 July 2019

26. Nguyen QD, Brown DM, Marcus DM et al (2012) Ranibizumab for diabetic macular edema: results from 2 phase III randomized trials: RISE and RIDE. Ophthalmology 119(4):789-801. https:// doi.org/10.1016/j.ophtha.2011.12.039

27. Brown DM, Nguyen QD, Marcus DM et al (2013) Long-term outcomes of ranibizumab therapy for diabetic macular edema: the 36-month results from two phase III trials: RISE and RIDE. Ophthalmology 120(10):2013-2022. https://doi.org/10.1016/j. ophtha.2013.02.034

28. European Medicines Agency (EMA) (2018) Eylea (aflibercept) summary of product characteristics. https://www.ema.europa.eu/ en/medicines/human/EPAR/eylea\#product-information-section. Accessed 13 July 2019

29. Brown DM, Schmidt-Erfurth U, Do DV et al (2015) Intravitreal aflibercept for diabetic macular edema: 100-week results from the VISTA and VIVID studies. Ophthalmology 122(10):2044-2052. https://doi.org/10.1016/j.ophtha.2015.06.017

30. Korobelnik JF, Do DV, Schmidt-Erfurth U et al (2014) Intravitreal aflibercept for diabetic macular edema. Ophthalmology 121(11):2247-2254. https://doi.org/10.1016/j.ophtha.2014.05.006

31. Mitchell P, Bandello F, Schmidt-Erfurth U et al (2011) The RESTORE study: ranibizumab monotherapy or combined with laser versus laser monotherapy for diabetic macular edema. Ophthalmology 118(4):615-625. https://doi.org/10.1016/j.ophth a.2011.01.031

32. European Medicines Agency (EMA) (2007) Lucentis (ranibizumab) EPAR scientific discussion 2007. https://www.ema.europ a.eu/en/documents/scientific-discussion/lucentis-epar-scientific -discussion_en.pdf. Accessed 13 July 2019

33. European Medicines Agency (EMA) (2013) Eylea EAR public assessment report 2013. https://www.ema.europa.eu/en/docum ents/assessment-report/eylea-epar-public-assessment-report_ en.pdf. Accessed 13 July 2019

34. Avery RL, Castellarin AA, Steinle NC et al (2017) Systemic pharmacokinetics and pharmacodynamics of intravitreal aflibercept, bevacizumab, and ranibizumab. Retina 37(10):1847-1858. https ://doi.org/10.1097/IAE.0000000000001493

35. Chen HX, Cleck JN (2009) Adverse effects of anticancer agents that target the VEGF pathway. Nat Rev Clin Oncol 6(8):465-477. https://doi.org/10.1038/nrclinonc.2009.94

36. Avery RL, Gordon GM (2016) Systemic safety of prolonged monthly anti-vascular endothelial growth factor therapy for diabetic macular edema: a systematic review and meta-analysis.
JAMA Ophthalmol 134(1):21-29. https://doi.org/10.1001/jamao phthalmol.2015.4070

37. Virgili G, Parravano M, Evans JR, Gordon I, Lucenteforte E (2017) Anti-vascular endothelial growth factor for diabetic macular oedema: a network meta-analysis. Cochrane Database Syst Rev 6(6):CD007419. https://doi.org/10.1002/14651858.cd007419. pub5

38. Peracha ZH, Rosenfeld PJ (2016) Anti-vascular endothelial growth factor therapy in pregnancy. Retina 36(8):1413-1417. https://doi. org/10.1097/IAE.0000000000001200

39. Concillado M, Lund-Andersen H, Mathiesen HR, Larsen M (2016) Dexamethasone intravitreal implant for diabetic macular edema during pregnancy. Am J Ophthalmol 165:7-15. https://doi. org/10.1016/j.ajo.2016.02.004

40. Romero-Aroca P, Baget-Bernaldiz M, Pareja-Rios A, Lopez-Galvez M, Navarro-Gil R, Verges R (2016) Diabetic macular edema pathophysiology: vasogenic versus inflammatory. J Diabetes Res 2016:2156273. https://doi.org/10.1155/2016/2156273

41. Boyer DS, Yoon YH, Belfort R Jr et al (2014) Three-year, randomized, sham-controlled trial of dexamethasone intravitreal implant in patients with diabetic macular edema. Ophthalmology 121(10):1904-1914. https://doi.org/10.1016/j.ophtha.2014.04.024

42. European Medicines Agency (EMA) (2018) Ozurdex (dexamethasone intravitreal implant) summary of product characteristics. https://www.ema.europa.eu/en/medicines/human/EPAR/ozurd ex\#product-information-section. Accessed 13 July 2019

43. Whitcup SM, Cidlowski JA, Csaky KG, Ambati J (2018) Pharmacology of corticosteroids for diabetic macular edema. Investig Ophthalmol Vis Sci 59(1):1-12. https://doi.org/10.1167/ iovs.17-22259

44. Chang-Lin JE, Attar M, Acheampong AA et al (2011) Pharmacokinetics and pharmacodynamics of a sustained-release dexamethasone intravitreal implant. Investig Ophthalmol Vis Sci 52(1):80-86. https://doi.org/10.1167/iovs.10-5285

45. Campochiaro PA, Hafiz G, Shah SM et al (2010) Sustained ocular delivery of fluocinolone acetonide by an intravitreal insert. Ophthalmology 117(7):1393-1399. https://doi.org/10.1016/j.ophth a.2009.11.024

46. Kiddee W, Trope GE, Sheng L et al (2013) Intraocular pressure monitoring post intravitreal steroids: a systematic review. Surv Ophthalmol 58(4):291-310. https://doi.org/10.1016/j.survophtha 1.2012.08.003

47. Thakur A, Kadam R, Kompella UB (2011) Trabecular meshwork and lens partitioning of corticosteroids: implications for elevated intraocular pressure and cataracts. Arch Ophthalmol 129(7):914920. https://doi.org/10.1001/archophthalmol.2011.39

48. Maturi RK, Pollack A, Uy HS et al (2016) Intraocular pressure in patients with diabetic macular edema treated with dexamethasone intravitreal implant in the 3-Year MEAD Study. Retina 36(6):1143-1152. https://doi.org/10.1097/IAE.000000000000100 4

49. Campochiaro PA, Brown DM, Pearson A et al (2012) Sustained delivery fluocinolone acetonide vitreous inserts provide benefit for at least 3 years in patients with diabetic macular edema. Ophthalmology 119(10):2125-2132. https://doi.org/10.1016/j.ophth a.2012.04.030

50. Hosseini K, Matsushima D, Johnson J et al (2008) Pharmacokinetic study of dexamethasone disodium phosphate using intravitreal, subconjunctival, and intravenous delivery routes in rabbits. J Ocul Pharmacol Ther 24(3):301-308. https://doi.org/10.1089/ jop. 2007.0117

51. Gan IM, Ugahary LC, van Dissel JT, van Meurs JC (2005) Effect of intravitreal dexamethasone on vitreous vancomycin concentrations in patients with suspected postoperative bacterial endophthalmitis. Graefes Arch Clin Exp Ophthalmol 243(11):1186-1189. https://doi.org/10.1007/s00417-005-1182-1 
52. Weijtens O, Feron EJ, Schoemaker RC et al (1999) High concentration of dexamethasone in aqueous and vitreous after subconjunctival injection. Am J Ophthalmol 128(2):192-197. https://doi. org/10.1016/s0002-9394(99)00129-4

53. Weijtens O, Schoemaker RC, Cohen AF et al (1998) Dexamethasone concentration in vitreous and serum after oral administration. Am J Ophthalmol 125(5):673-679. https://doi.org/10.1016/s0002 -9394(98)00003-8

54. Weijtens O, Schoemaker RC, Romijn FP, Cohen AF, Lentjes EG, van Meurs JC (2002) Intraocular penetration and systemic absorption after topical application of dexamethasone disodium phosphate. Ophthalmology 109(10):1887-1891

55. Weijtens O, van der Sluijs FA, Schoemaker RC et al (1997) Peribulbar corticosteroid injection: vitreal and serum concentrations after dexamethasone disodium phosphate injection. Am J Ophthalmol 123(3):358-363. https://doi.org/10.1016/s0002 -9394(14)70131-x

56. Bucolo C, Gozzo L, Longo L, Mansueto S, Vitale DC, Drago F (2018) Long-term efficacy and safety profile of multiple injections of intravitreal dexamethasone implant to manage diabetic macular edema: a systematic review of real-world studies. J Pharmacol Sci 138(4):219-232. https://doi.org/10.1016/j.jphs.2018.11.001

57. Rajesh B, Zarranz-Ventura J, Fung AT et al (2019) Safety of 6000 intravitreal dexamethasone implants. Br J Ophthalmol. https://doi. org/10.1136/bjophthalmol-2019-313991

58. Moisseiev E, Loewenstein A (2017) Diabetic macular edema: emerging strategies and treatment algorithms. Dev Ophthalmol 60:165-174. https://doi.org/10.1159/000459706

59. Callanan DG, Loewenstein A, Patel SS et al (2017) A multicenter, 12-month randomized study comparing dexamethasone intravitreal implant with ranibizumab in patients with diabetic macular edema. Graefes Arch Clin Exp Ophthalmol 255(3):463-473. https ://doi.org/10.1007/s00417-016-3472-1

60. Busch C, Zur D, Fraser-Bell S et al (2018) Shall we stay, or shall we switch? Continued anti-VEGF therapy versus early switch to dexamethasone implant in refractory diabetic macular edema. Acta Diabetol 55(8):789-796. https://doi.org/10.1007/s0059 2-018-1151-x

61. Vermeire E, Wens J, Van Royen P, Biot Y, Hearnshaw H, Lindenmeyer A (2005) Interventions for improving adherence to treatment recommendations in people with type 2 diabetes mellitus. Cochrane Database Syst Rev 2(2):CD003638. https://doi. org/10.1002/14651858.cd003638.pub2

62. Claxton AJ, Cramer J, Pierce C (2001) A systematic review of the associations between dose regimens and medication compliance. Clin Ther 23(8):1296-1310

63. Kruk ME, Schwalbe N (2006) The relation between intermittent dosing and adherence: preliminary insights. Clin Ther 28(12):1989-1995. https://doi.org/10.1016/j.clint hera.2006.12.011

64. Querques L, Parravano M, Sacconi R, Rabiolo A, Bandello F, Querques $G$ (2017) Ischemic index changes in diabetic retinopathy after intravitreal dexamethasone implant using ultra-widefield fluorescein angiography: a pilot study. Acta Diabetol 54(8):769-773. https://doi.org/10.1007/s00592-017-1010-1

65. Iglicki M, Zur D, Busch C, Okada M, Loewenstein A (2018) Progression of diabetic retinopathy severity after treatment with dexamethasone implant: a 24-month cohort study the 'DR-Pro-DEX Study'. Acta Diabetol 55(6):541-547. https://doi.org/10.1007/ s00592-018-1117-z

66. Antiplatelet Trialists' Collaboration (1994) Collaborative overview of randomised trials of antiplatelet therapy-I: prevention of death, myocardial infarction, and stroke by prolonged antiplatelet therapy in various categories of patients. BMJ 308(6921):81-106

Publisher's Note Springer Nature remains neutral with regard to jurisdictional claims in published maps and institutional affiliations. 